\title{
Effects of spin-orbit coupling on the electron-phonon superconductivity in the cubic Laves-phase compounds $\mathrm{CaIr}_{2}$ and $\mathrm{CaRh}_{2}$
}

\author{
H. M. Tütüncü, ${ }^{1,2}$ H. Y. Uzunok,,${ }^{1,2}$ Ertuğrul Karaca, ${ }^{2}$ E. Arslan, ${ }^{1}$ and G. P. Srivastava ${ }^{3}$ \\ ${ }^{1}$ Fen-Edebiyat Fakültesi, Fizik Bölümü, Sakarya Üniversitesi, 54187, Adapazarı, Turkey \\ ${ }^{2}$ BIMAYAM Biyomedikal, Manyetik ve Yarililetken Malzemeler Araştırma Merkezi, Sakarya Üniversitesi, 54187, Adapazarı, Turkey \\ ${ }^{3}$ School of Physics, University of Exeter, Stocker Road, Exeter EX4 4QL, United Kingdom \\ (Received 3 July 2017; revised manuscript received 6 October 2017; published 16 October 2017)
}

\begin{abstract}
We report our ab initio pseudopotential results for the structural, electronic, vibrational, and electron-phonon interaction properties of the cubic Laves-phase compounds $\mathrm{CaIr}_{2}$ and $\mathrm{CaRh}_{2}$. While the spin-orbit coupling (SOC) does not result in any appreciable changes in structural parameters, it lifts the degeneracies of some bands near the Fermi level, albeit with a much smaller amount for $\mathrm{CaRh}_{2}$. The effect of SOC on the vibrational properties of both materials is considerable. The SOC results in a slight decrease in the electronic density of states near the Fermi level $N\left(E_{F}\right)$ and makes low-frequency phonon branches harder, and the electron-phonon coupling parameter $\lambda$ is lowered from 1.43 to 1.05 for $\mathrm{CaIr}_{2}$ and from 1.17 to 0.96 for $\mathrm{CaRh}_{2}$. On the other hand, the logarithmically averaged phonon frequency $\omega_{\ln }$ is enhanced from 79.60 to $100.97 \mathrm{~K}$ for $\mathrm{CaIr}_{2}$ and from 120.20 to $140.80 \mathrm{~K}$ for $\mathrm{CaRh}_{2}$ with the inclusion of SOC. Using the calculated values of $\lambda$ and $\omega_{\mathrm{ln}}$, the superconducting critical temperature is determined to be $5.94 \mathrm{~K}\left(7.34 \mathrm{~K}\right.$ without SOC) for $\mathrm{CaIr}_{2}$ and $6.97 \mathrm{~K}\left(9.08 \mathrm{~K}\right.$ without SOC) for $\mathrm{CaRh}_{2}$. The superconducting critical-temperature values with SOC compare very well with corresponding experimental values of 5.80 and $6.40 \mathrm{~K}$, indicating the importance of SOC for the physical properties of both materials.
\end{abstract}

DOI: 10.1103/PhysRevB.96.134514

\section{INTRODUCTION}

$\mathrm{AB}_{2}$-type compounds with Laves-phase structures have received a great deal of attention from the scientific community because these compounds can be considered magnetic [1-8] and hydrogen storage materials [9-23]. Laves-phase compounds have also been widely used in hightemperature structural applications because of their high melting temperatures, low densities, and high-oxidation resistances [24-27]. Furthermore, Laves phases are very important materials for the electronic and spacecraft industries due to their interesting physical properties $[28,29]$. $\mathrm{AB}_{2}$-type Laves-phase compounds belong to the intermetallics family, where $\mathrm{A}$ is an electropositive metal, such as an alkali, alkaline earth, lanthanide, actinide, or early transition metal, and the B material is a less electropositive transition or noble metal. The Laves phase is categorized primarily into three crystal structures: the $\mathrm{MgCu}_{2} C 15$-type cubic structure of the space group $F d \overline{3} m, \mathrm{MgZn}_{2} C$ 14-type hexagonal structure of the space group $P 6_{3} / m m c$ and $\mathrm{MgNi}_{2}$ C36-type dihexagonal structure of the space group $P 6_{3} / \mathrm{mmc}$. Experimental studies [30,31] showed that the $C 36$-type structure is an intermediate phase between the other two phases.

Among these three phases, the $C 15$ one is expected to display much better deformability than the other two phases because the independent slip systems are permitted in its fcc-based structure [32]. Several $C 15$ compounds are known as superconductors [33-43]. In 2015, the speculated superconducting parameters determined from magnetization and specific-heat measurements revealed that $\mathrm{CaIr}_{2}$ exhibits superconductivity with the transition temperature of $5.8 \mathrm{~K}$ [41]. This experimental work [41] appears to be interesting because electron correlations and spin-orbit interactions are very important for designating the electronic, magnetic, and superconducting properties of compounds based on $5 d$ transition metals [44-59]. Haldolaarachchige and coworkers [41] have also presented the electronic properties of the cubic Laves-phase compound $\mathrm{CaIr}_{2}$. This theoretical work revealed that the density of states at the Fermi level $N\left(E_{F}\right)$ is mainly contributed by Ir $5 d$ states [41]. Although the effect of spin-orbit interaction (SOI) on the electronic properties of $\mathrm{CaIr}_{2}$ [41] has been investigated, the effect of this interaction on its phonon-related properties is still lacking in the literature. However, the lattice dynamics of this material must be studied because, when studying systems in the metallic state, many physical properties, such as electrical and thermal resistivity, thermal expansion, and superconductivity, seem to be specified by phonons and their interactions with electrons. In a pioneering work on phonon-mediated electron-pairing interaction, Heid et al. [60] found that the inclusion of SOI leads to an increase in the electron-phonon coupling constant via phonon softening in $\mathrm{Pb}$. The work by Heid et al. further shows that, in contrast to $\mathrm{Pb}$, there are only weak effects of SOI on the phonon spectrum and electron-phonon interaction in the neighboring element $\mathrm{Tl}$. Thus, in order to reveal the origin of superconductivity and, in particular, to clarify the effect of SOI in the cubic Laves-phase compounds $\mathrm{CaIr}_{2}$ and $\mathrm{CaRh}_{2}$ within the conventional BCS [61] theory of superconductivity, we have to study their electronic, vibrational, and electronphonon-interaction properties.

In this work, we present and discuss the structural and electronic properties of the cubic Laves-phase $\mathrm{CaIr}_{2}$ and $\mathrm{CaRh}_{2}$ using the $a b$ initio pseudopotential method based on a generalized gradient approximation (GGA) of density functional theory with and without spin-orbit coupling (SOC). Phonons in both materials were studied using a linear response theory $[62,63]$. The electron-phonon matrix elements for both compounds were evaluated by using the linear response method [62,63] and the Migdal-Eliashberg approach [64,65]. Then, the Eliashberg spectral functions for these compounds were calculated using their phonon density of states and 
their electron-phonon matrix elements. The average electronphonon coupling parameter and the logarithmic average of phonon frequency for both materials are determined by integrating their Eliashberg spectral functions. Using the electronphonon coupling parameter and the logarithmic average of phonon frequency, we have computed the superconducting transition temperatures $T_{c}$. The effect of including SOC on these properties is analyzed and quantified.

\section{THEORY}

Within the Migdal-Eliashberg theory of superconductivity $[64,65]$, first-principles calculations [62,63] of superconducting properties need knowledge of (i) the electronic structure, (ii) the phonon spectrum, and (iii) the electron-phonon matrix elements of the material. The first-principles calculations presented in this work were using the QUANTUM ESPRESSO program [62], which is based on the density functional theory, within the plane-wave pseudopotential method. We use fully relativistic ultrasoft pseudopotentials [66] to model the electron-ion interactions. The Perdew-Burke-Ernzerhof GGA [67] functional is used to solve the Kohn-Sham equations [68]. The energy convergence is checked with respect to the plane-wave kinetic-energy cutoff and $\boldsymbol{k}$-point sampling. A $(12 \times 12 \times 12)$ zone-centered grid is adopted to determine the structural parameters of both materials. For electronic calculations, a denser $(24 \times 24 \times 24) \boldsymbol{k}$-point grid is adopted.

Following self-consistent solutions of the Kohn-Sham equations, the lattice dynamical properties (the phonon spectrum, the density of states, and the eigenvectors corresponding to phonon frequencies) are determined within the framework of the self-consistent density functional perturbation theory [62]. We calculate eight dynamical matrices for a $4 \times 4 \times 4$ $\boldsymbol{q}$-point mesh within the irreducible part of the Brillouin zone. Then, these dynamical matrices are Fourier transformed to calculate phonons for any chosen $\boldsymbol{q}$ point. The technique to calculate the electron-phonon coupling can be found in previous $a b$ initio studies [57,63]. A $(24 \times 24 \times 24) k$-point mesh is chosen to ensure $\boldsymbol{k}$-point sampling convergence with Gaussians with a width of $0.03 \mathrm{Ry}$, which approximates the zero-width limits in the calculations of the phonon linewidth and electron-phonon coupling parameter.

\section{RESULTS}

\section{A. Structural and electronic properties}

Both studied intermetallic compounds crystallize in the cubic Laves-phase $\mathrm{AB}_{2}(C 15)$ crystal structure with space
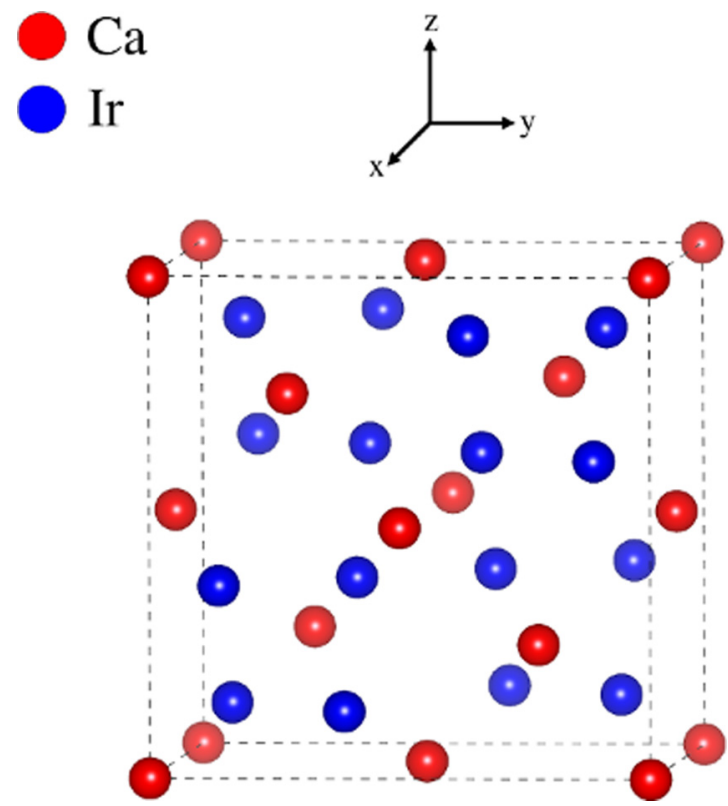

FIG. 1. The cubic Laves crystal structure of $\mathrm{CaIr}_{2}$ and $\mathrm{CaRh}_{2}$.

group $F d \overline{3} m$. The conventional cubic unit cell includes eight molecules of the $\mathrm{AB}_{2}$ as illustrated in Fig. 1. The $\mathrm{A}$ atoms are arranged on the diamond structure sites, and the $\mathrm{B}$ atoms generate tetrahedra. Thus, the nearest-neighbor $A$ distance is $\frac{\sqrt{3} a}{4}$, while the nearest-neighbor B distance is $\frac{\sqrt{2} a}{4}$. The positions of the basis of six atoms (two $\mathrm{AB}_{2}$ molecules) of the $\mathrm{AB}_{2}$ type related to the primitive unit cell given in terms of the lattice parameter $a$ are $(0,0,0)$ and $(1 / 4,1 / 4,1 / 4)$ for $\mathrm{A}$ and $(5 / 8,5 / 8,5 / 8),(5 / 8,7 / 8,7 / 8)$, $(7 / 8,5 / 8,7 / 8)$, and $(7 / 8,7 / 8,5 / 8)$ for $B$. Thus, the structure information is completely defined by the lattice parameter $a$. The lattice parameter, the bulk modulus $B$, and its pressure derivative $B^{\prime}$ are evaluated by fitting the total energy as a function of the lattice parameter to the Murnaghan equation of states [69]. The calculated values for the equilibrium lattice parameter $a$, the equilibrium volume $V$, the bulk modulus $B$, its pressure derivative $B^{\prime}$, and the equilibrium bond lengths are presented in Table I. This table shows clearly that the spin-orbit interaction has only a negligible effect on the structural properties of both materials. Thus, we will use the lattice parameters with SOC for further calculations. Since the total energy calculations have been made using the GGA functional, the calculated values of lattice

TABLE I. Structural parameters $\left(a, B\right.$, and $\left.B^{\prime}\right)$ and bond lengths $\left(d_{C a-C a}, d_{C a-M}\right.$, and $\left.d_{M-M}\right)$ with and without SOC for CaM $2(M=\mathrm{Ir}$ or $\mathrm{Rh}$ ) in the cubic Laves structure and their comparison with experimental results.

\begin{tabular}{|c|c|c|c|c|c|c|c|}
\hline Material & $a(\AA)$ & $V\left(\AA^{3}\right)$ & $d_{C a-C a}(\AA)$ & $d_{C a-M}(\AA)$ & $d_{M-M}(\AA)$ & $B(\mathrm{GPa})$ & $B^{\prime}$ \\
\hline $\mathrm{CaIr}_{2}$ with SOC & 7.595 & 109.53 & 3.288 & 3.149 & 2.685 & 162.6 & 4.77 \\
\hline $\mathrm{CaIr}_{2}$ without SOC & 7.594 & 109.48 & 3.288 & 3.148 & 2.685 & 166.0 & 4.77 \\
\hline Experiment [33] & 7.545 & 107.38 & 3.267 & 3.128 & 2.667 & & \\
\hline $\mathrm{CaRh}_{2}$ with SOC & 7.585 & 109.09 & 3.284 & 3.145 & 2.682 & 122.8 & 4.65 \\
\hline $\mathrm{CaRh}_{2}$ without SOC & 7.581 & 108.92 & 3.283 & 3.143 & 2.680 & 123.6 & 4.67 \\
\hline Experiment [33] & 7.525 & 106.53 & 3.258 & 3.119 & 2.661 & & \\
\hline
\end{tabular}




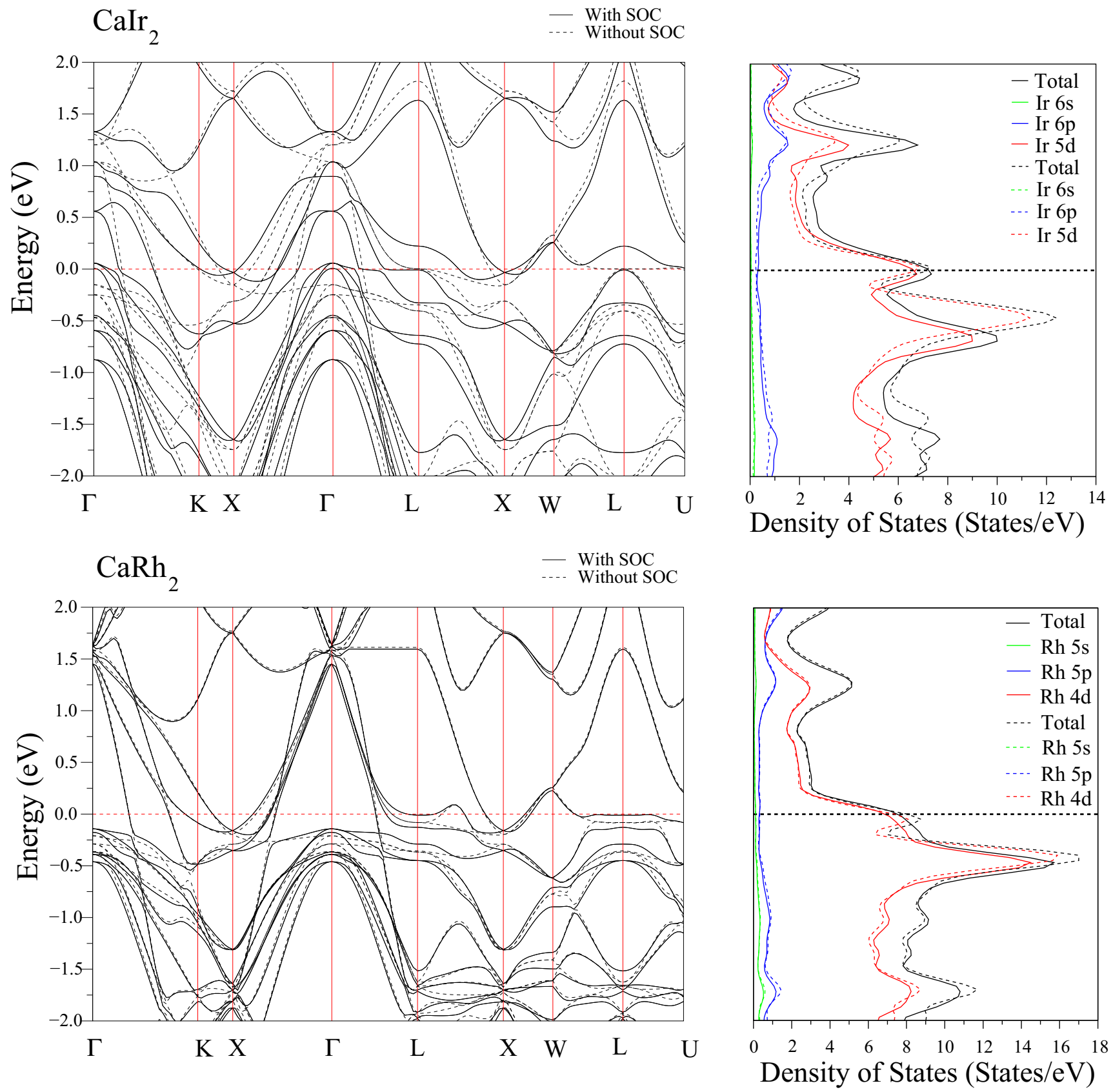

FIG. 2. The calculated electronic band structures and electronic density of states for $\mathrm{CaIr}_{2}$ and $\mathrm{CaRh}_{2}$ with and without spin-orbit coupling (SOC). The inclusion of SOC has split several bands.

parameters are slightly overestimated by approximately $0.7 \%$ and $0.8 \%$ for $\mathrm{CaIr}_{2}$ and $\mathrm{CaRh}_{2}$, respectively, compared to their experimental values listed in Table I. Unfortunately, there are no experimental data for $B$ and $B^{\prime}$ for us to compare with. Although not shown in Fig. 1, Ca and Ir (Rh) atoms have coordination numbers of 16 and 12 , respectively. Three types of chemical bonds occur in this structure: $\mathrm{Ca}-\mathrm{Ca}$, Ir-Ir (or $\mathrm{Rh}-\mathrm{Rh}$ ), and $\mathrm{Ca}-\mathrm{Ir}$ (or $\mathrm{Ca}-\mathrm{Rh}$ ) bonds. As can be seen from Table I, our computed chemical bond lengths agree very well with experimental values [33]. The calculated bond lengths Ca-Ca $(3.288 \AA)$, Ir-Ir (2.685 $)$, and Ca-Ir (3.149 $\AA$ ) are smaller than the simple sum of the covalent radii for each atom, implying that these bonds have a significant covalent character.
A similar observation has been made for the corresponding chemical bonds in $\mathrm{CaRh}_{2}$.

Spin-orbit interaction in an atom is due to the interaction of electronic spin moment with the magnetic field formed by the traveling electron in the electric field of the nucleus. Since the nuclear charge enhances with the rise in atomic number of the elements, heavier atoms are characterized by larger SOC strength. The band structure and electronic density of states presented in Fig. 2 for $\mathrm{CaIr}_{2}$ agree well with a previous theoretical calculation [41]. Along the high-symmetry directions $\Gamma-K-X-\Gamma-L$, the bands are more dispersive and cross the Fermi level, which confirms the metallic nature of $\mathrm{CaIr}_{2}$. The valence bands around the high-symmetry $\Gamma$ point display 

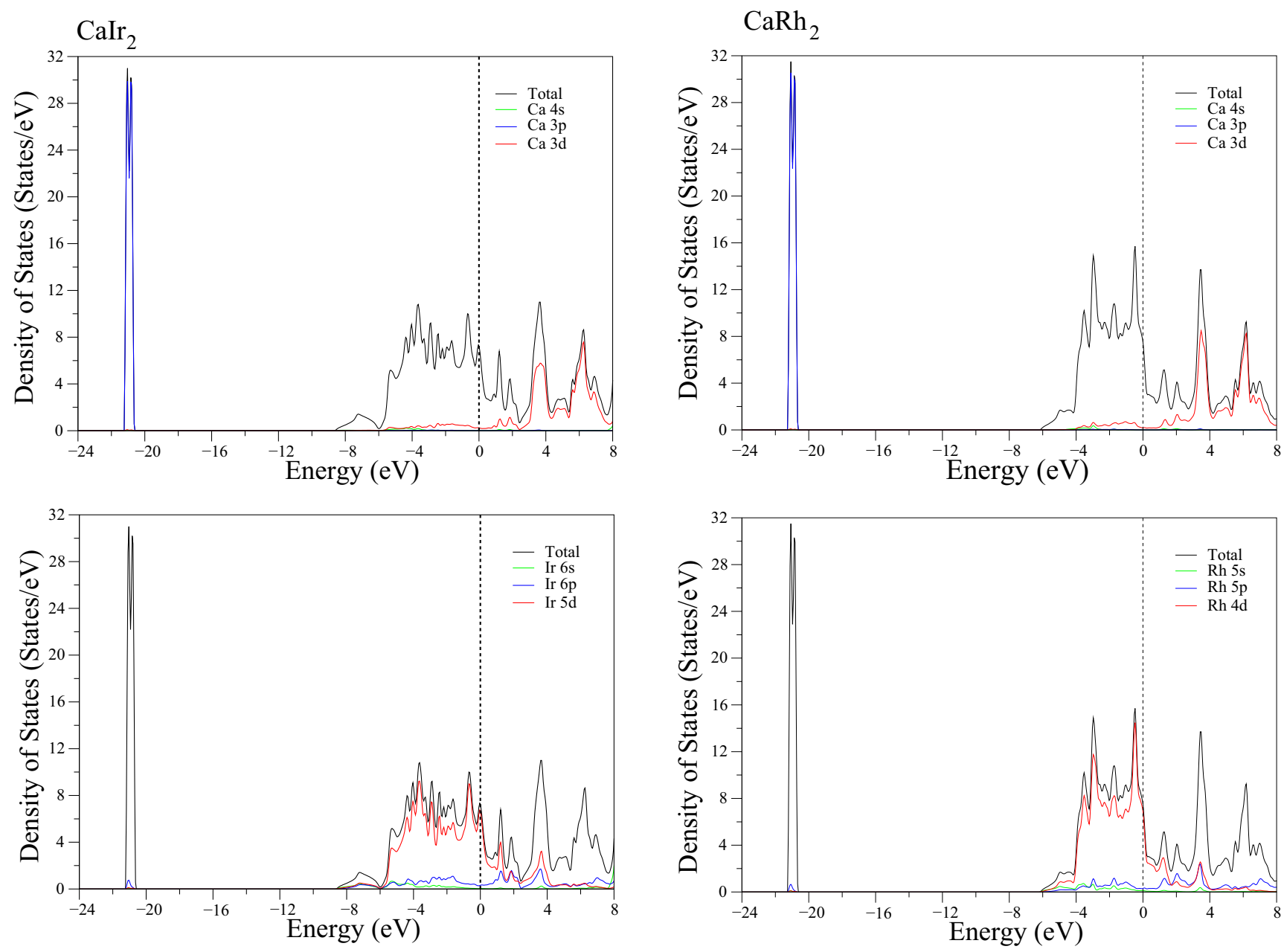

FIG. 3. The calculated total and partial electronic densities of states of $\mathrm{CaIr}_{2}$ and $\mathrm{CaRh}_{2}$ with SOC.

explicit relativistic effects. SOC gives rise to the splitting of degenerate bands at the $\Gamma$ point and modifies the outline of the bands.

In order to find out the origin of electronic bands in the electronic structures of $\mathrm{CaIr}_{2}$ and $\mathrm{CaRh}_{2}$, we have evaluated and presented their total and partial densities of states (DOSs) with SOC in Fig. 3. At first glance, the DOSs of both compounds look similar to each other. However, when the Ir atom is replaced by a $\mathrm{Rh}$ atom, the main valence-band region in $\mathrm{CaRh}_{2}$ is shortened from 8.6 to $6.0 \mathrm{eV}$ because of the replacement of the Ir $5 d$ states by the $\mathrm{Rh} 4 d$ states. The contribution of the valence states of the $\mathrm{Ca}$ atom to the main valence region for both materials is small and featureless since this atom is almost in the form of cation $\mathrm{Ca}^{2+}$ and behaves like electronic donors. Thus, the effect of the $\mathrm{Ca}$ atom on the electronic and superconducting properties of both compounds can be ignored due to the fact that the Ca-like DOS around $E_{F}$ is negligible. For both compounds, the majority of transition-metal $d$ states appears between -6.0 and $0.0 \mathrm{eV}$ and hybridizes weakly with $\mathrm{Ca} d$ and transition-metal $s$ and $p$ states. Thus, transition-metal $d$-like states dominate the DOS features close to Fermi level for both materials. The density of states at the Fermi level $N\left(E_{F}\right)$ with SOC for $\mathrm{CaIr}_{2}$ amounts to 6.72 states $/ \mathrm{eV}$, which is comparable to the value of 6.98 states/eV estimated from the heat-capacity data [41].
$\mathrm{Ca}$ and $\mathrm{Ir}$ electronic states make a contribution to $N\left(E_{F}\right)$ within around $4 \%$ and $96 \%$. In particular, $91 \%$ of $N\left(E_{F}\right)$ is contributed by only Ir $d$ states. The value of $N\left(E_{F}\right)$ with $\mathrm{SOC}$ for $\mathrm{CaRh}_{2}$ is found to be 7.09 states $/ \mathrm{eV}$, which is higher than the corresponding value for $\mathrm{CaIr}_{2}$. The value of $N\left(E_{F}\right)$ for the $d$ states of $\mathrm{Rh}$ atom is calculated to be 6.90 states $/ \mathrm{eV}$, which is higher than the corresponding value of 6.45 states $/ \mathrm{eV}$ for the $d$ states of the Ir atom. Thus, the total increase in the value of $N\left(E_{F}\right)$ for $\mathrm{CaRh}_{2}$ can be connected to an increase in the contribution from the $d$ states of the $\mathrm{Rh}$ atom. As a result, the huge contribution from transition-metal $d$ states to $N\left(E_{F}\right)$ for both materials can be suggested to have a strong influence on superconductivity in both materials since Cooper pairs in the BCS theory are formed by electrons with energies around the Fermi level. In general, the spin-orbit splitting of degenerate bands increases as the fourth power of nuclear charge $Z^{4}$. From our work we find that the value of $N\left(E_{F}\right)$ is slightly reduced when SOC is incorporated. However, this reduction is a little more pronounced in $\mathrm{CaIr}_{2}$, which is associated with the $5 d$ orbital in the Ir atom.

\section{B. Electron-phonon interaction}

Six atoms in the primitive unit cell of the cubic Laves phase of $\mathrm{CaIr}_{2}$ and $\mathrm{CaRh}_{2}$ give rise to 3 acoustic and 15 optical 
TABLE II. Calculated frequencies of zone-center phonon modes $v$ (in $\mathrm{THz}$ ) and eigencharacters for $\mathrm{CaIr}_{2}$ and $\mathrm{CaRh}_{2} . \mathrm{S}$, IR, and $\mathrm{R}$ indicate silent, infrared-active, and Raman-active vibrations, respectively.

\begin{tabular}{lcccccc}
\hline \hline Mode & $E_{u}$ & $T_{1 u}$ & $T_{2 u}$ & $T_{1 u}$ & $A_{2 u}$ & $T_{2 g}$ \\
\hline \multicolumn{7}{c}{$\mathrm{CaIr}_{2}$} \\
$v$ with SOC & 1.68 & 2.55 & 2.69 & 5.52 & 6.58 & 6.91 \\
$v$ without SOC & 1.73 & 1.95 & 2.56 & 5.44 & 6.60 & 6.92 \\
Eigencharacters & $\mathrm{Ir}$ & $\mathrm{Ir}+\mathrm{Ca}$ & $\mathrm{Ir}$ & $\mathrm{Ca}+\mathrm{Ir}$ & $\mathrm{Ir}$ & $\mathrm{Ca}$ \\
Active & $\mathrm{S}$ & $\mathrm{IR}$ & $\mathrm{S}$ & $\mathrm{IR}$ & $\mathrm{S}$ & $\mathrm{R}$ \\
& \multicolumn{7}{c}{$\mathrm{CaRh}_{2}$} & & & \\
$v$ with SOC & 2.86 & 3.49 & 2.66 & 5.41 & 7.71 & 6.48 \\
$v$ without SOC & 2.80 & 3.10 & 2.54 & 5.26 & 7.67 & 6.58 \\
Eigencharacters & $\mathrm{Rh}$ & $\mathrm{Rh}+\mathrm{Ca}$ & $\mathrm{Rh}$ & $\mathrm{Ca}+\mathrm{Rh}$ & $\mathrm{Rh}$ & $\mathrm{Ca}$ \\
Active & $\mathrm{S}$ & $\mathrm{IR}$ & $\mathrm{S}$ & $\mathrm{IR}$ & $\mathrm{S}$ & $\mathrm{R}$ \\
\hline \hline
\end{tabular}

phonon branches. We first examine the zone-center phonon modes, as these can be measured by different experimental techniques. The zone-center optical phonon modes of both materials can be categorized by the irreducible presentation of the point group $O_{h}(m \overline{3} m)$ :

$$
\Gamma\left(O_{h}\right)=E_{u}+2 T_{1 u}+T_{2 u}+A_{2 u}+T_{2 g},
$$

with the $A, E$, and $T$ modes being singly, doubly, and triply degenerate, respectively. The frequencies of these zone-center optical phonon modes for both materials are presented in Table II. Of the triply degenerate modes, the $T_{2 g}$ mode is Raman active (R), while the $T_{1 u}$ mode is infrared (IR) active. However, the remaining modes are optically silent (S). In order to understand the effect of SOC on the vibrational modes at the zone center, we have also included our results without SOC for both materials. Table II clearly shows that the effect of SOC on the zone-center phonon frequencies is considerable. In particular, the effect of this interaction on the zone-center phonon modes of $\mathrm{CaIr}_{2}$ is much stronger than those of $\mathrm{CaRh}_{2}$. This can be linked to the heavier mass of the Ir atom compared to that of the Rh atom because, as stated earlier, the strength of the SOC effect is proportional to $Z^{4}$. Upon inclusion of SOC, the lowest $T_{1 u}$ phonon mode of $\mathrm{CaIr}_{2}$ gets harder by about $24 \%$. This strong change will affect the electron-phonon coupling parameter $\lambda$ of this phonon mode. When SOC is taken into account, the value of $\lambda$ for this phonon mode decreases from 0.13 to 0.09 . Thus, we can conclude that phonons in both these materials must be studied with SOC taken into account.

Accurate determination of electron-phonon coupling requires understanding of the full phonon dispersion curves through the Brillouin zone. Figure 4 illustrates the phonon dispersion curves of both materials along high-symmetry directions in the Brillouin zone of a fcc lattice with and without SOC. At the calculated equilibrium volumes of $\mathrm{CaIr}_{2}$ and $\mathrm{CaRh}_{2}$, all phonon modes have positive frequencies, indicating that both these materials are dynamically stable in their cubic $\mathrm{MgCu}_{2}$ structure. The phonon branches fill the entire frequency range of both materials, leaving no gap in their phonon dispersion curves. The phonon spectrum of $\mathrm{CaIr}_{2}$ spans up to about 7.1 THz, while that of $\mathrm{CaRh}_{2}$ is extended by about $0.6 \mathrm{THz}$ mainly due to the smaller mass of the $\mathrm{Rh}$ atom compared to the mass of the Ir atom. Low-frequency optical
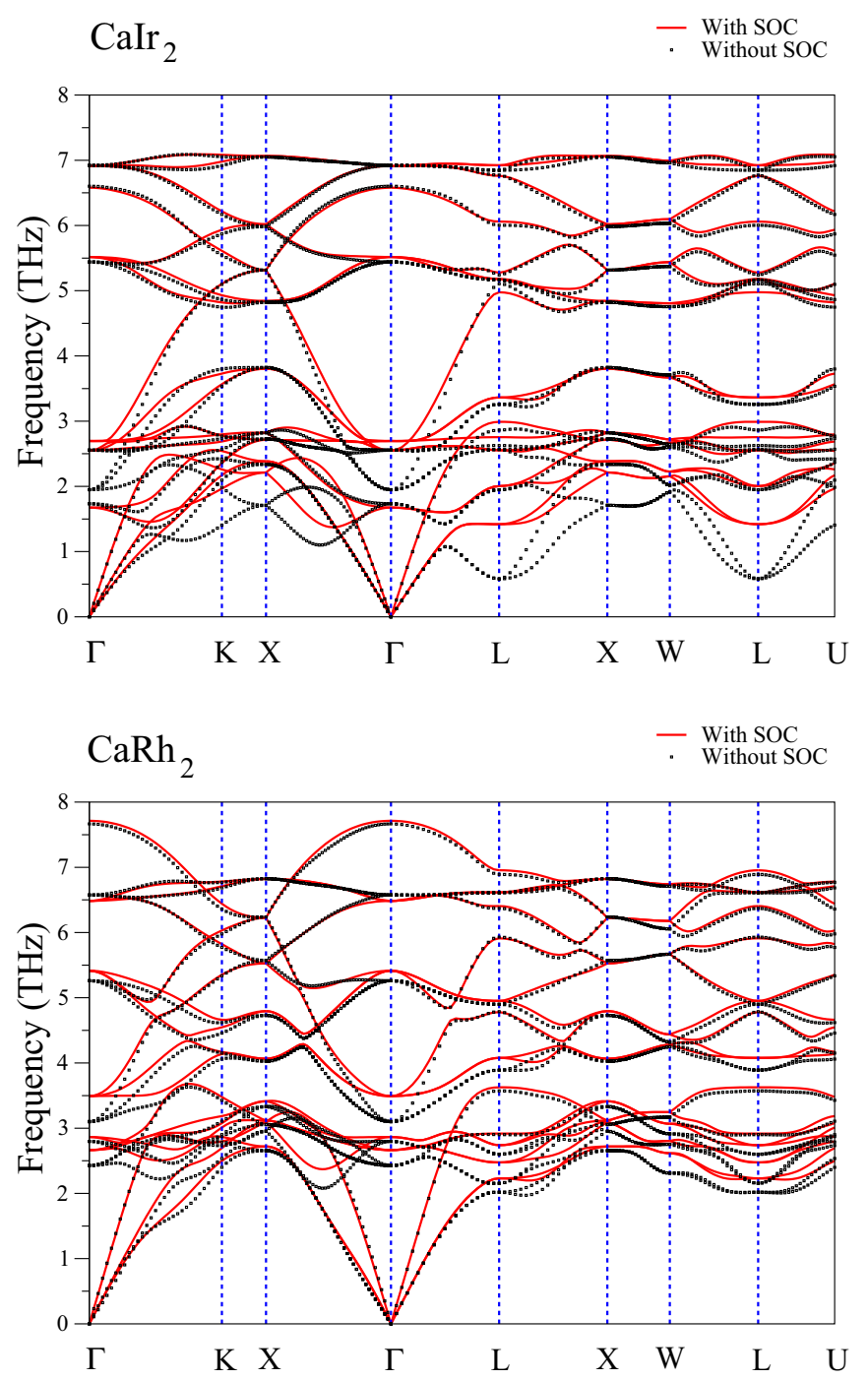

FIG. 4. The calculated phonon dispersion curves of $\mathrm{CaIr}_{2}$ and $\mathrm{CaRh}_{2}$ with and without SOC.

phonon modes and acoustic phonon modes of both materials exhibit significant dispersive character, with considerable overlap between these phonon branches. We have to mention that upon inclusion of SOC, these phonon branches of $\mathrm{CaIr}_{2}$ become much harder. However, the hardening of these phonon modes with $\mathrm{SOC}$ for $\mathrm{CaRh}_{2}$ is much smaller but considerable nevertheless.

The contributions of atomic vibrations to phonon branches can be much better understood by analyzing the total and partial phonon DOSs. Total and partial densities of states for $\mathrm{CaIr}_{2}$ are displayed in Fig. 5. The DOS features below 3.8 $\mathrm{THz}$ originate from the vibrations of the heavier Ir atoms with a lesser contribution from the vibrations of lighter $\mathrm{Ca}$ atoms. The frequency region from 3.8 to $4.7 \mathrm{THz}$ is derived from the coupled motion of both atoms. The frequency region above 4.7 THz exhibits a dominance of the lighter $\mathrm{Ca}$ atoms. Although Ir atoms are much heavier than $\mathrm{Ca}$ atoms, they still make a visible contribution to the DOS features between 4.7 and 6.6 THz. This contribution confirms the existence of a strong covalent bonding force between $\mathrm{Ca}$ and $\mathrm{Ir}$ atoms. Upon 

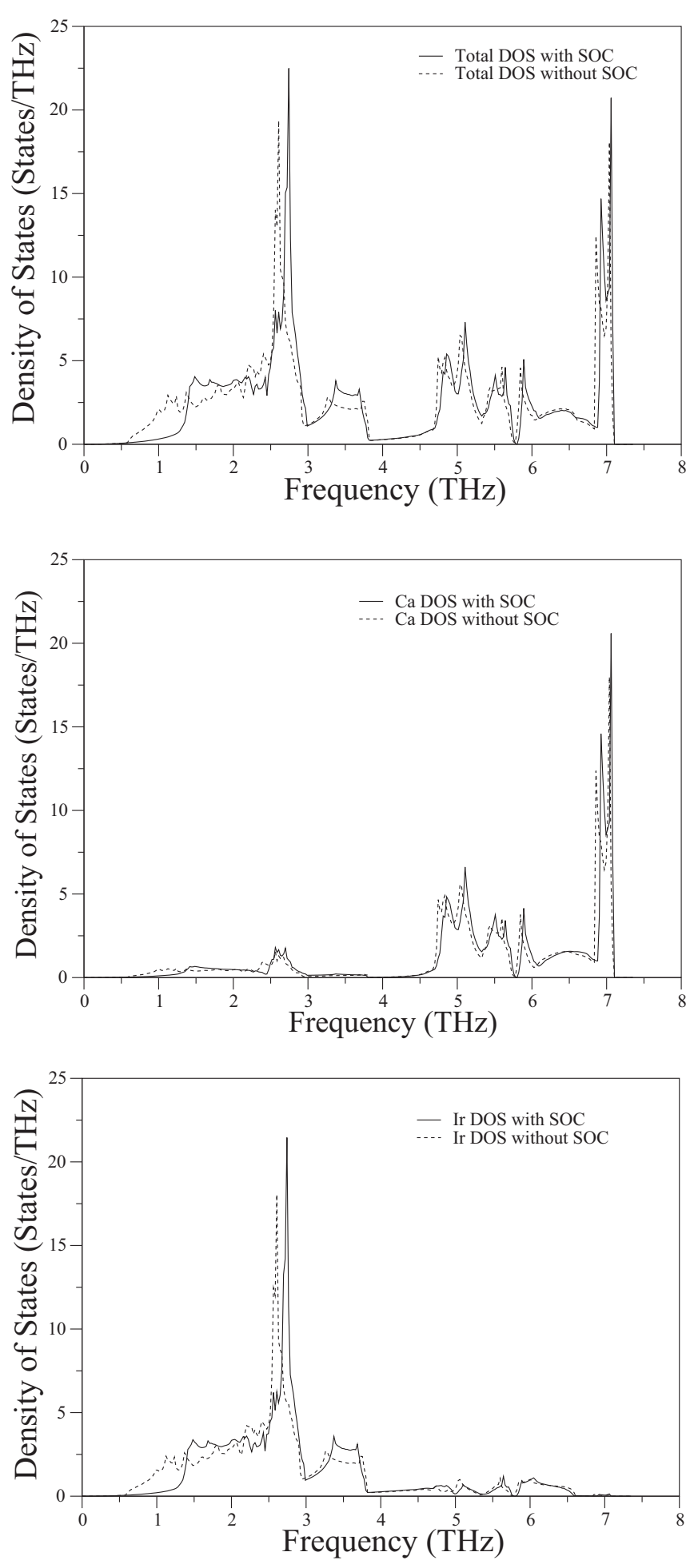

FIG. 5. The calculated total and atom-projected phonon densities of states of $\mathrm{CaIr}_{2}$ with and without SOC.

inclusion of SOC, the Ir-related phonon states below $3.0 \mathrm{THz}$ are considerably shifted up. This change certainly affects the average electron-phonon coupling parameter $\lambda$ of $\mathrm{CaIr}_{2}$ because this parameter is essentially connected to the phonon density of states through the Eliashberg spectral function $\left[\alpha^{2} F(\omega)=\alpha^{2}(\omega) F(\omega)\right][64,65] . \alpha^{2}(\omega)$ contains an average square electron-phonon matrix elements, while $F(\omega)$ is similar to the phonon density of states. From the calculated $\alpha^{2} F(\omega)$, one can evaluate the electron-phonon coupling parameter $\lambda$ by $[64,65]$

$$
\lambda=2 \int \frac{\alpha^{2}(\omega) F(\omega)}{\omega} d \omega .
$$

This equation provides the most reliable value for $\lambda$. The electron-phonon coupling parameter $\lambda$ can also be given in the form

$$
\lambda=\frac{N\left(E_{F}\right)\left\langle I^{2}\right\rangle}{M\left\langle\omega^{2}\right\rangle},
$$

where $\left\langle I^{2}\right\rangle$ represents the Fermi surface average of squared electron-phonon coupling interaction and $M$ and $\left\langle\omega^{2}\right\rangle$ are the mass of the atoms and the average of squared phonon frequencies. The above McMillan-Hopfield expression suggests that the electron-phonon coupling parameter will considerably decrease when phonon modes harden. As a consequence, it is expected to obtain a smaller electron-phonon coupling parameter when the effect of SOC is considered.

The total and partial phonon densities of states for $\mathrm{CaRh}_{2}$ are displayed in Fig. 6. The phonon DOS of $\mathrm{CaRh}_{2}$ is similar to that of $\mathrm{CaIr}_{2}$. Our computational work shows that despite no changes in structural parameters, the incorporation of SOC results in an overall increase in the transition-metal-related force constants. In particular, we find that the Ir-Ir and Rh-Rh self-terms increase by $10.9 \%$ and $10.6 \%$, respectively. Such changes in the interatomic force constants can be related to the SOC-governed changes in the electronic states related to the transition metals in these systems. Consequently, in general, the effect of SOC is to shift peaks in the DOS to higher frequencies. This is more appreciable in the low-frequency regime (typically, below $3 \mathrm{THz}$ ). We also find that the DOS in the lower acoustic region has been reduced. However, different from $\mathrm{CaIr}_{2}, \mathrm{Rh}$ atoms in $\mathrm{CaRh}_{2}$ partake in lattice vibrations over the whole range of phonon frequencies, and $\mathrm{Ca}$ vibrations almost disappear above $7.0 \mathrm{THz}$. The contribution of Rh to the phonon modes is strongest below $3.7 \mathrm{THz}$, while the partial DOS displays a dominance of $\mathrm{Ca}$ atoms in the frequency region between 4.3 and $7.0 \mathrm{THz}$. Finally, a strong overlap between $\mathrm{Rh}$ and $\mathrm{Ca}$ vibrations exists between 3.7 and $4.3 \mathrm{THz}$ due to the strong covalent bonding between these atoms. Once again, the frequencies of transition-metal-related phonon modes are increased when the SOC is taken into account. Thus, we again expect that the electron-phonon coupling parameter for $\mathrm{CaRh}_{2}$ will acquire a smaller value when SOC is included.

To identify the strengths with which various kinds of vibrational modes interact with electrons and thus affect the superconducting properties, the Eliashberg spectral function $\alpha^{2} F(\omega)$ is computed for both materials. The electron-phonon spectral function $\alpha^{2} F(\omega)$ and the average electron-phonon coupling parameter $\lambda$ for both materials with and without SOC are illustrated in Fig. 7. The results with and without SOC for both materials differ from each other in the lowfrequency region (typically, below $3.8 \mathrm{THz}$ ). Such a difference in $\alpha^{2} F(\omega)$ clearly indicates that SOC plays an important role in determining electron-phonon interaction in both materials. Inclusion of SOC reduces electron-phonon coupling for both materials due to the hardening of lower phonon modes. The 

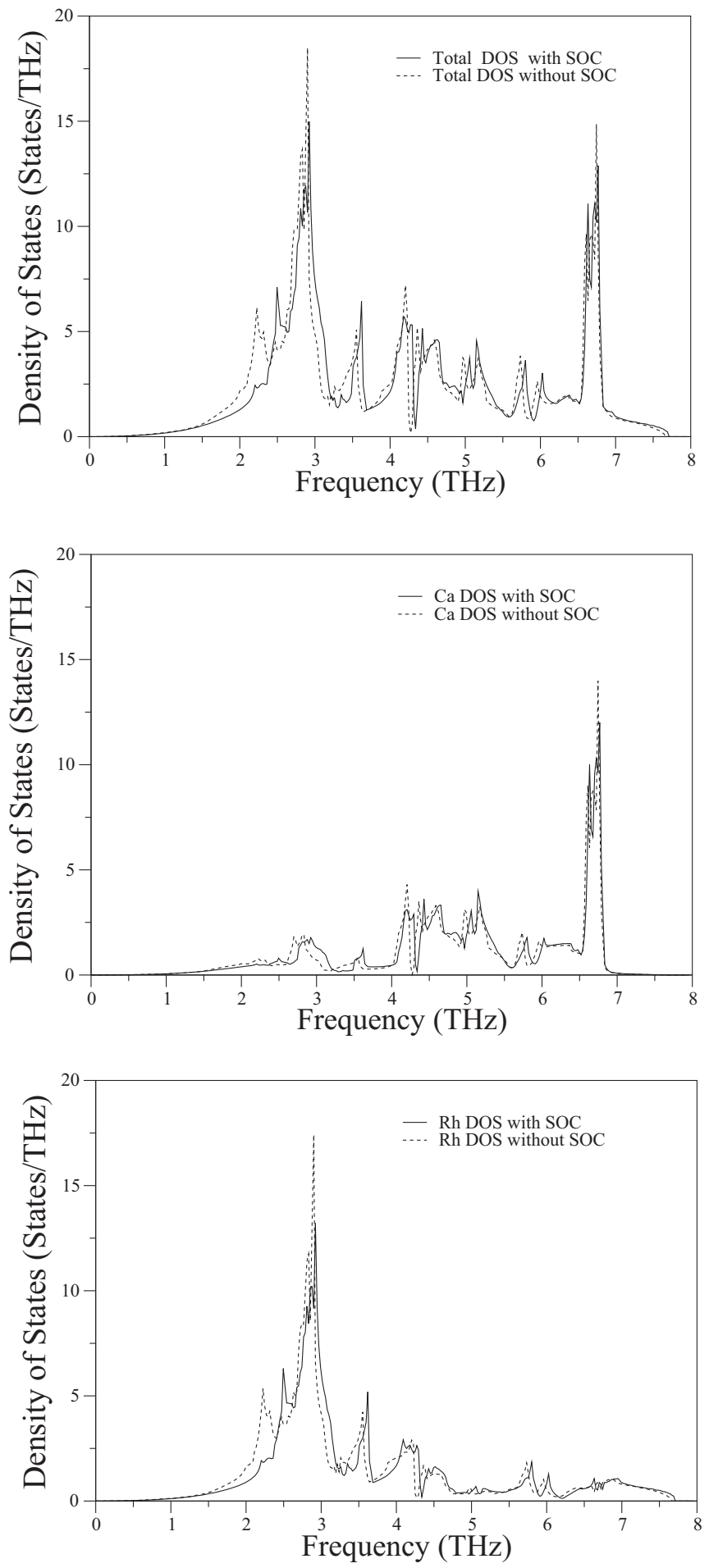

FIG. 6. The calculated total and atom-projected phonon densities of states of $\mathrm{CaRh}_{2}$ with and without SOC.

value of $\lambda$ without SOC is found to be 1.43 and 1.17 for $\mathrm{CaIr}_{2}$ and $\mathrm{CaRh}_{2}$. These values decrease to 1.05 and 0.96 , respectively, when the SOC is considered. In the frequency region below $3.8 \mathrm{THz}, \lambda$ for both materials increases with increasing frequency. The phonon modes in this region contribute about $96 \%$ (1.008) to $\lambda$ for $\mathrm{CaIr}_{2}$ and about $93 \%$ (0.884) for $\mathrm{CaRh}_{2}$. These huge contributions are expected because

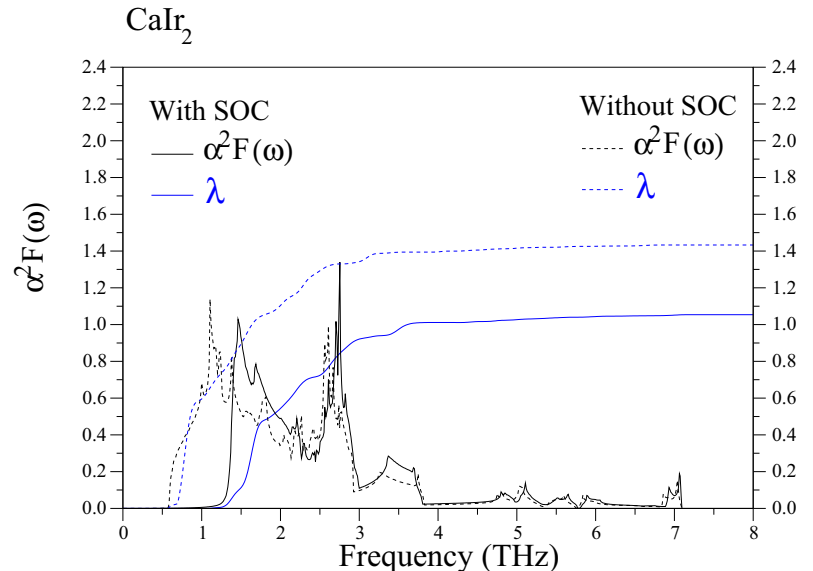

$\lambda$

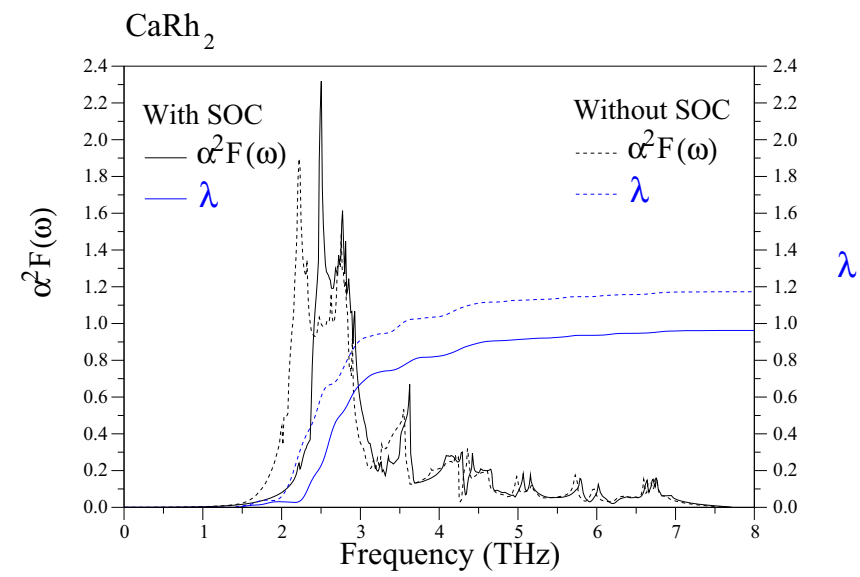

FIG. 7. Eliashberg spectral function $\alpha^{2} F(\omega)$ (solid line) and integrated electron-phonon coupling parameter $\lambda$ (dashed line) for $\mathrm{CaIr}_{2}$ and $\mathrm{CaRh}_{2}$.

the vibrations of transition-metal atoms may bring about strong electron-phonon interaction because of the considerable existence of transition-metal $d$ electrons at the Fermi level. On the other hand, the contribution of high-frequency phonon modes (above $3.8 \mathrm{THz}$ ) to $\lambda$ is about $4 \%(0.042)$ for $\mathrm{CaIr}_{2}$ and about $7 \%(0.076)$ for $\mathrm{CaRh}_{2}$. Thus, we can conclude the motion of the $\mathrm{Ca}$ atom plays a relatively insignificant role in determining the superconducting properties of both materials. It is useful at this stage to mention that the presently obtained trend of the SOI effect of reduction in $\lambda$ via phonon hardening in the cubic $\mathrm{CaIr}_{2}$ and $\mathrm{CaRh}_{2}$ should not be taken as a general feature for both materials. This can be appreciated by comparing our finding with the pioneering work by Heid et al. [60], who obtained an increase in $\lambda$ via phonon softening [shift to lower frequencies and increased weight of the peaks in $\alpha^{2} F(\omega)$ to promote an increase in $\lambda$ ]. Further work on other systems may help us understand if there is a general trend of the effect of SOI in determining BCS-type superconducting features of materials.

Using the average electron-phonon coupling constant $\lambda$ and the logarithmically averaged phonon frequency $\omega_{\ln }[64,65]$,

$$
\omega_{l n}=\exp \left(2 \lambda^{-1} \int_{0}^{\infty} \frac{d \omega}{\omega} \alpha^{2} F(\omega) \ln \omega\right)
$$


TABLE III. Calculated electron density of states at the Fermi level $N\left(E_{F}\right)$, average electron-phonon coupling parameter $\lambda$, logarithmically averaged phonon frequency $\omega_{l n}$, and superconducting critical temperature $T_{c}$. Experimental results for the value of the superconducting critical temperature $T_{c}$ are also presented for comparison.

\begin{tabular}{lcccc}
\hline \hline Material & $N\left(E_{F}\right)($ states $/ \mathrm{eV})$ & $\lambda$ & $\omega_{l n}(\mathrm{~K})$ & $T_{c}(\mathrm{~K})$ \\
\hline $\mathrm{CaIr}_{2}$ with SOC & 6.73 & 1.05 & 100.97 & 5.94 \\
$\mathrm{CaIr}_{2}$ without SOC & 6.52 & 1.43 & 79.600 & 7.34 \\
Experiment [35] & & & & 6.15 \\
Experiment [41] & 6.98 & & & 5.8 \\
$\mathrm{CaRh}_{2}$ with SOC & 7.09 & 0.96 & 140.80 & 6.97 \\
$\mathrm{CaRh}_{2}$ without SOC & 7.21 & 1.17 & 120.20 & 9.08 \\
Experiment [35] & & & & 6.40 \\
\hline \hline
\end{tabular}

the superconducting transition temperature $T_{c}$ is computed from the Allen-Dynes modification of the McMillan formula $[64,65]$

$$
T_{C}=\frac{\omega_{\ln }}{1.2} \exp \left(-\frac{1.04(1+\lambda)}{\lambda-\mu^{*}(1+0.62 \lambda)}\right),
$$

where $\mu^{*}$ is an effective screened Coulomb repulsion constant which usually takes value between 0.10 and $0.16[64,65]$. In this work, the value of $\mu^{*}$ is fixed to be 0.15 following the experimental work of Haldolaarachchige and coworkers [41]. The calculated parameters $\left[N\left(E_{F}\right), \lambda, \omega_{\mathrm{ln}}\right.$, and $\left.T_{c}\right]$ connected to superconductivity in both materials are presented in Table III. As can be seen from this table, inclusion of SOC results in an increase of the value of $\omega_{\ln }$ for both materials due to the hardening of lower phonon modes. This change makes the value of $\lambda$ smaller for both materials because $\lambda$ decreases with increasing phonon frequencies according to the McMillanHopfield expression [see also Eq. (2)]. Consequently, when SOC is taken into account, the value of $T_{c}$ for both materials is lowered from 7.34 to $5.94 \mathrm{~K}$ for $\mathrm{CaIr}_{2}$ and from 9.08 to $6.97 \mathrm{~K}$ for $\mathrm{CaRh}_{2}$. The $T_{c}$ values with $\mathrm{SOC}$ agree very well with their experimental values of 5.80 and $6.40 \mathrm{~K}[35,41]$. This good agreement suggests that the physical properties of both materials must be studied while considering SOC.

\section{SUMMARY}

We have presented $a b$ initio pseudopotential results for the structural, electronic, phononic, and electron-phonon interaction properties of $\mathrm{CaIr}_{2}$ and $\mathrm{CaRh}_{2}$ crystallizing in the cubic Laves-type structure with and without SOC. The electronic band structures of both materials are very similar to each other, but the splitting effects due to SOC on $\mathrm{CaIr}_{2}$ are more significant because of the larger effective atomic number of the Ir atom compared to that of the Rh atom. Our electronic results suggest that $\mathrm{Ca}$ electronic states make an insignificant contribution to the main valence-band region of both materials, while their transition-metal $d$ states dominate this region. This finding confirms that superconductivity in these materials is associated with their transition-metal atoms, while the role of the $\mathrm{Ca}$ atom is to stabilize their structure. Our structural and electronic results are utilized to investigate the vibrational properties of both materials using a linear response approach based on the density functional theory. Upon inclusion of SOC, low-frequency phonon branches of $\mathrm{CaIr}_{2}$ become much harder, while the hardening of these phonon branches with SOC for $\mathrm{CaRh}_{2}$ is much smaller but noticeable. Thus, a significant SOC effect on the phonon dispersion curves of both materials is associated with their transition-metal atoms, whose vibrations dominate the formation of these low-frequency phonon modes.

The Eliashberg spectral function for both materials is evaluated from their calculated phonon spectrum and their calculated electron-phonon matrix elements. The spectral functions with and without SOC for both materials differ from each other in the low-frequency region below $3.8 \mathrm{THz}$ since including SOC leads to a reduction in electron-phonon interaction for both materials due to the hardening of lower phonon modes. When SOC is included, these phonon modes become harder, and thus, the electron-phonon coupling parameter for both materials is lowered. This decrease is from 1.43 to 1.05 for $\mathrm{CaIr}_{2}$ and from 1.17 to 0.96 for $\mathrm{CaRh}_{2}$. All these values indicate that the electron-phonon interaction in both materials is strong. The calculated Eliashberg spectral function for both materials with and without SOC reveals that the lowfrequency phonon modes are strongly involved in the process of scattering electrons near the Fermi level. These phonon branches contribute to the average electron-phonon coupling parameter $\lambda$ by more than $90 \%$. This result is expected because these phonon modes arise from the vibrations of transition-metal atoms, and their $d$ states make the largest contribution to the density of states at the Fermi level. Finally, using the Allen-Dynes modified McMillan equation with the screened Coulomb pseudopotential parameter $\mu^{*}=0.15$, the superconducting critical temperature is determined to be $5.94 \mathrm{~K}$ (7.34 K without SOC) for $\mathrm{CaIr}_{2}$ and $6.97 \mathrm{~K}$ (9.08 K without SOC) for $\mathrm{CaRh}_{2}$. The superconducting critical-temperature values with SOC are in good agreement with their experimental values of 5.80 and $6.40 \mathrm{~K}$, respectively. These results reveal that SOC must be considered correctly for determining physical properties of both materials. Finally, we hope that our investigations will stimulate further experimental and theoretical works on the cubic Laves phase and related phases.

\section{ACKNOWLEDGMENT}

Some of the calculations for this project were carried out using the computing facilities on the Intel Nehalem (i7) cluster (ceres) in the School of Physics, University of Exeter, United Kingdom.
[1] M. B. Moffett, A. E. Clark, F. M. Wun, J. Linderberg, J. B. Teter, and E. A. Mclaughlin, J. Acoust. Soc. Am. 89, 1448 (1991).

[2] Ch. V Mohan, J. Alloys Compd. 238, 86 (1996).
[3] Z. Wu, N. L. Saini, S. I. Agrestin, D. D. Castro, A. Bianconi, A. Marcelli, M. Battisti, D. Gozzi, and G. Balducci, J. Phys. Condens. Matter 12, 6971 (2000). 
[4] H. Uchida, Y. Matsumura, H. Uchida, and H. Kaneko, J. Magn. Mater. 239, 540 (2002).

[5] X.-Q. Chen, W. Wolf, R. Podloucky, P. Rogl, and M. Marsman, Phys. Rev. B 72, 054440 (2005).

[6] X.-Q. Chen, W. Wolf, R. Podloucky, and P. Rogl, Phys. Rev. B 76, 014424 (2007).

[7] N. K. Singh, K. G. Suresh, A. K. Nigam, S. K. Malik, A. A. Coelho, and S. Gama, J. Magn. Magn. Mater. 317, 68 (2007).

[8] R. M. Shabara and S. H. Aly, J. Magn. Magn. Mater. 423, 447 (2017).

[9] S. L. Hong and C. L. Fu, Phys. Rev. B 66, 094109 (2002).

[10] N. Nagasako, A. Fukumoto, and K. Miwa, Phys. Rev. B 66, 155106 (2002).

[11] A. V. Skripov, T. J. Udovic, and J. J. Rush, Phys. Rev. B 76, 104305 (2007).

[12] M. Kandavel, V. V. Bhat, A. Rougier, L. Aymard, G.-A. Nazri, and J.-M. Tarascon, Int. J. Hydrogen Energy 33, 3754 (2008).

[13] G. Srinivas, V. Sankaranarayanan, and S. Ramaprabhu, J. Alloys Compd. 448, 159 (2008).

[14] X. Liu, K. Asano, N. Terashita, and E. Akiba, Int. J. Hydrogen Energy 34, 1472 (2009).

[15] S. B. Gesari, M. E. Pronsato, A. Visintin, and A. Juan, J. Phys. Chem. C 114, 16832 (2010).

[16] X. W. Yang, T. B. Zhang, R. Hu, J. S. Li, X. Y. Xue, and H. Z. Fu, Int. J. Hydrogen Energy 35, 11981 (2010).

[17] J. Ahmad, H. Maekawa, H. Takamura, I. Oikawa, M. Ando, and K. Takahashi, J. Alloys Compd. 540, 222 (2012).

[18] K. Young, M. Young, T. Ouchi, B. Reichman, and M. A. Fetcenko, J. Power Sources 215, 279 (2012).

[19] D. F. Wong, K. Young, J. Nei, L. Wang, and K. Y. S. Ng, J. Alloys Compd. 647, 507 (2015).

[20] T. D. Wu, X. Y. Xue, T. B. Zhang, Y. L. Zhang, H. C. Kou, and J. S. Li, Int. J. Hydrogen Energy 41, 19114 (2016).

[21] S. Banerjee, A. Kumar, P. Ruz, and P. Sengupta, Int. J. Hydrogen Energy 41, 18130 (2016).

[22] T. B. Zhang, T. D. Wu, X. Y. Xue, R. Hu, H. C. Kou, and J. S. Li, Renewable Energy 103, 786 (2017).

[23] L. Rabahi, M. Gallouze, T. Grosdidier, D. Bradai, and A. Kellou, Int. J. Hydrogen Energy 42, 2157 (2017).

[24] A. Von Keitz and G. Sauthoff, Intermetallics 10, 497 (2002).

[25] D. J. Thoma, K. A. Nibur, and K. C. Chen, Mater. Sci. Eng. A 329-331, 408 (2002).

[26] J. J. Liu, W. J. Ren, Z. D. Zhang, D. Li, J. Li, X. G. Zhao, W. Liu, and S. W. Or, J. Appl. Phys. 100, 023904 (2006).

[27] X. W. Nie, S. Q. Lu, and K. L. Wang, Powder Technol. 184, 333 (2008).

[28] K. Turba, P. Ma'lek, and M. Cieslar, Mater. Sci. Eng. A 462, 91 (2007).

[29] A. Kumar, A. K. Mukhopadhyay, and K. S. Prasad, Mater. Sci. Eng. A 527, 854 (2010).

[30] J. D. Thoma and J. H. Perepezko, Mater. Sci. Eng. A 156, 97 (1992).

[31] S. Hong and C. L. Fu, Intermetallics 7, 5 (1999).

[32] A. Ormeci, F. Chu, J. M. Wills, T. E. Mitchell, R. C. Albers, D. J. Thoma, and S. P. Chen, Phys. Rev. B 54, 12753 (1996).

[33] E. A. Wood and V. B. Compton, Acta Crystallogr. 11, 429 (1958).

[34] V. B. Compton and B. T. Matthias, Acta Crystallogr. 12, 651 (1959).

[35] B. T. Matthias, T. H. Geballe, and V. B. Compton, Rev. Mod. Phys. 35, 1 (1963).
[36] T. F. Smith and H. L. Luo, J. Phys. Chem. Solids 28, 569 (1967).

[37] J. F. Cannon, D. L. Robertson, H. T. Hall, and A. C. Lawson, J. Less-Common Met. 31, 174 (1973).

[38] R. N. Shelton, A. C. Lawson, and K. Baberschke, Solid State Commun. 24, 465 (1977).

[39] V. A. Finkel and E. A. Pushkarev, Zh. Eksp. Teor. Fiz. 78, 842 (1980) [Sov. Phys. JETP 51, 422 (1980)].

[40] H. Takei, M. Yamawaki, A. Oota, and S. Noguchi, J. Phys. F 15, 2333 (1985).

[41] N. Haldolaarachchige, Q. Gibson, L. M. Schoop, H. Luo, and R. J. Cava, J. Phys. Condens. Matter 27, 185701 (2015).

[42] S. Sun, K. Liu, and H. Lei, J. Phys. Condens. Matter 28, 085701 (2016).

[43] K. W. Chen, D. Graf, T. Besara, A. Gallagher, N. Kikugawa, L. Balicas, T. Siegrist, A. Shekhter, and R. E. Baumbach, Phys. Rev. B 93, 045118 (2016).

[44] Y. Singh and S. Ramakrishnan, Phys. Rev. B 69, 174423 (2004).

[45] E. Bauer, R. T. Khan, H. Michor, E. Royanian, A. Grytsiv, N. Melnychenko-Koblyuk, P. Rogl, D. Reith, R. Podloucky, E.-W. Scheidt, W. Wolf, and M. Marsman, Phys. Rev. B 80, 064504 (2009).

[46] N. H. Sung, C. J. Roh, K. S. Kim, and B. K. Cho, Phys. Rev. B 86, 224507 (2012).

[47] S. Pyon, K. Kudo, and M. Nohara, J. Phys. Soc. Jpn. 81, 053701 (2012).

[48] T. Takayama, K. Kuwano, D. Hirai, Y. Katsura, A. Yamamoto, and H. Takagi, Phys. Rev. Lett. 108, 237001 (2012).

[49] F. Kneidinger, H. Michor, A. Sidorenko, E. Bauer, I. Zeiringer, P. Rogl, C. Blaas-Schenner, D. Reith, and R. Podloucky, Phys. Rev. B 88, 104508 (2013).

[50] R. Khasanov, A. Amato, P. K. Biswas, H. Luetkens, N. D. Zhigadlo, and B. Batlogg, Phys. Rev. B 90, 140507(R) (2014).

[51] S. Pyon, K. Kudo, J. Matsumura, H. Ishii, G. Matsuo, M. Nohara, H. Hojo, K. Oka, M. Azuma, O. Garlea, K. Kodama, and S. Shamoto, J. Phys. Soc. Jpn. 83, 093706 (2014).

[52] M. Smidman, A. D. Hillier, D. T. Adroja, M. R. Lees, V. K. Anand, R. P. Singh, R. I. Smith, D. M. Paul, and G. Balakrishnan, Phys. Rev. B 89, 094509 (2014).

[53] T. Shiroka, M. Pikulski, N. D. Zhigadlo, B. Batlogg, J. Mesot, and H.-R. Ott, Phys. Rev. B 91, 245143 (2015).

[54] H. Y. Lu, N. N. Wang, L. Geng, S. Chen, Y. Yang, W. J. Lu, W. S. Wang, and J. Sun, Europhys. Lett. 110, 17003 (2015).

[55] J. F. Landaeta, S. V. Taylor, I. Bonalde, C. Rojas, Y. Nishikubo, K. Kudo, and M. Nohara, Phys. Rev. B 93, 064504 (2016).

[56] K. Hu, B. Gao, Q. Ji, Y. Ma, W. Li, X. Xu, H. Zhang, G. Mu, F. Huang, C. Cai, X. Xie, and M. Jiang, Phys. Rev. B 93, 214510 (2016).

[57] A. S. Cuamba, H.-Y. Lu, and C. S. Ting, Phys. Rev. B 94, 094513 (2016).

[58] H. Y. Uzunok, E. İpsara, H. M. Tütüncü, G. P. Srivastava, and A. Başoglu, J. Alloys Compd. 681, 205 (2016).

[59] H. Y. Uzunok, H. M. Tütüncü, G. P. Srivastava, and A. Başoglu, Intermetallics 86, 1 (2017).

[60] R. Heid, K.-P. Bohnen, I. Yu. Sklyadneva, and E. V. Chulkov, Phys. Rev. B 81, 174527 (2010).

[61] J. Bardeen, L. N. Cooper, and J. R. Schrieffer, Phys. Rev. 108, 1175 (1957).

[62] P. Giannozzi, S. Baroni, N. Bonini, M. Calandra, R. Car, C. Cavazzoni, D. Ceresoli, G. L. Chiarotti, M. Cococcioni, I. Dabo, A. D. Corso, S. de Gironcoli, S. Fabris, G. Fratesi, 
R. Gebauer, U. Gerstmann, C. Gougoussis, A. Kokalj, M. Lazzeri, L. Martin-Samos, N. Marzari, F. Mauri, R. Mazzarello, S. Paolini, A. Pasquarello, L. Paulatto, C. Sbraccia, S. Scandolo, G. Sclauzero, A. P. Seitsonen, A. Smogunov, P. Umari, and R. M. Wentzcovitch, J. Phys. Condens. Matter 21, 395502 (2009).

[63] H. M. Tütüncü, H. Y. Uzunok, E. Karaca, G. P. Srivastava, S. Özer, and Ş. Uğur, Phys. Rev. B 92, 054510 (2015).

[64] P. B. Allen, Phys. Rev. B 6, 2577 (1972).
[65] P. B. Allen and R. C. Dynes, Phys. Rev. B 12, 905 (1975).

[66] A. M. Rappe, K. M. Rabe, E. Kaxiras, and J. D. Joannopoulos, Phys. Rev. B 41, 1227 (1990).

[67] J. P. Perdew, K. Burke, and M. Ernzerhof, Phys. Rev. Lett. 77, 3865 (1996).

[68] W. Kohn and L. J. Sham, Phys. Rev. 140, A1133 (1965).

[69] F. D. Murnaghan, Proc. Natl. Acad. Sci. USA 50, 697 (1944). 